\title{
Spinning branes in Riemann-Cartan spacetime
}

\author{
Milovan Vasilid* and Marko Vojinovid \\ Institute of Physics, P.O.Box 57, 11001 Belgrade, Serbia
}

(Dated: December 28, 2018)

\begin{abstract}
We use the conservation law of the stress-energy and spin tensors to study the motion of massive brane-like objects in Riemann-Cartan geometry. The world-sheet equations and boundary conditions are obtained in a manifestly covariant form. In the particle case, the resultant world-line equations turn out to exhibit a novel spin-curvature coupling. In particular, the spin of a zero-size particle does not couple to the background curvature. In the string case, the world-sheet dynamics is studied for some special choices of spin and torsion. As a result, the known coupling to the Kalb-Ramond antisymmetric external field is obtained. Geometrically, the Kalb-Ramond field has been recognized as a part of the torsion itself, rather than the torsion potential.
\end{abstract}

PACS numbers: 04.40.-b

\section{INTRODUCTION}

The problem of motion of brane like objects in backgrounds of nontrivial geometry is addressed by using some form of the Mathisson-Papapetrou method [1, 2]. One starts with the covariant conservation law of the stress-energy and spin tensors of matter fields, and analyzes it under the assumption that matter is localized to resemble a brane. In the lowest, single-pole approximation, the moving matter is viewed as an infinitely thin brane. In the pole-dipole approximation, its non-zero thickness is taken into account.

The known results concerning extended objects in Riemann-Cartan geometry exclusively refer to particles. They can be summarized as follows. Spinless particles in the single-pole approximation obey the geodesic equation. In the pole-dipole approximation, the rotational angular momentum of the localized matter couples to spacetime curvature, and produces geodesic deviation [15]. If the particles have spin, the curvature couples to the total angular momentum, and the torsion to the spin alone [6-10].

As for the higher branes, the results found in literature exclusively refer to spinless matter and Riemannian backgrounds [11, 12]. It has been shown that spacetime curvature couples to the internal angular momentum of a thick brane, and that this coupling disappears if the brane is infinitely thin.

In this paper, we shall study spinning brane-like matter in spacetimes with curvature and torsion. Our motivation is threefold. First, realistic strings (like flux tubes) are really believed to exist, and to be relevant for the description of hadronic matter. Second, we want to check if the presence of matter with spin saves the spin-curvature coupling even if the brane has no thickness. Finally, the influence of torsion on the brane dynamics can provide a geometric insight into the extended string actions found

*Electronic address: mvasilic@phy.bg.ac.yu

${ }^{\dagger}$ Electronic address: vmarko@phy.bg.ac.yu in literature. Namely, the basic Nambu-Goto string action [13, 14] is in literature often modified to include interaction with additional background fields. Apart from the target-space metric, the antisymmetric tensor field $\mathcal{B}_{\mu \nu}(x)$ and the dilaton field $\Phi(x)$ are considered [15 18]. While spacetime metric has obvious geometric interpretation, the background fields $\mathcal{B}_{\mu \nu}(x)$ and $\Phi(x)$ do not. The attempts have been made in literature to interpret $\mathcal{B}_{\mu \nu}$ and $\Phi$ as originating from the background torsion and non-metricity, respectively [19 25]. Our idea is to consider stringy shaped matter in backgrounds of general geometry, and check if the effective action of Refs. 15-18] could be recovered. This way, the real geometric nature of the background fields $\mathcal{B}_{\mu \nu}(x)$ and $\Phi(x)$ could be found.

The results that we have obtained are summarized as follows. The world-sheet equations and boundary conditions for a $p$-dimensional brane in $D$-dimensional Riemann-Cartan spacetime are derived in a manifestly covariant way. It has been shown that spacetime curvature couples to $(p+1)$-dimensional currents associated with the internal angular momentum of the brane, while torsion couples to the spin alone. The curvature coupling disappears in the limit of an infinitely thin brane, in spite of the fact that brane is made of spinning matter. As illustrative examples, the 0-brane (particle) and 1-brane (string) are given an additional consideration. The particle dynamics has been found to differ from what has been believed so far. In particular, the spin of a strict point particle does not couple to the curvature. We have also analyzed the world-sheet equations and boundary conditions of an infinitely thin string. The generalized string action of Refs. 15 18] has been recovered by assigning a special value to the spin tensor and the background torsion. According to our results, the Kalb-Ramond antisymmetric tensor field $\mathcal{B}_{\mu \nu}(x)$ is related to the torsion itself, rather than to its potential as suggested in literature.

The layout of the paper is as follows. In section II, we define the conservation law of the stress-energy and spin tensors, and introduce the necessary geometric notions. Using the fact that antisymmetric part of the 
stress-energy tensor is completely determined by the spin tensor, we eliminate it from further considerations. The conservation equations are rewritten in terms of the independent variables - the spin tensor and the generalized Belinfante tensor. After the brief recapitulation of the covariant multipole formalism, we define the pole-dipole approximation for the independent variables, only. Section IIII is devoted to the derivation of the brane worldsheet equations. The actual derivation is only sketched, as the method has already been analyzed in detail in [12]. The world-sheet equations and boundary conditions are obtained in a manifestly covariant form. In Section IV particles and strings are given additional consideration. In the particle case, the resulting equations of motion are compared to the pole-dipole equations found in literature [8, 9]. As it turns out, they coincide in the pole-dipole approximation, but have different single-pole limits. In the string case, the world-sheet equations are analyzed in the zero-thickness limit. By an appropriate choice of the spin-tensor and the background torsion, we have recovered the effective dynamics of Refs. 15 18]. In section V] we give our final remarks.

Our conventions are the same as in Ref. [12]. Greek indices $\mu, \nu, \ldots$ are the spacetime indices, and run over $0,1, \ldots, D-1$. Latin indices $a, b, \ldots$ are the worldsheet indices and run over $0,1, \ldots, p$. The Latin indices $i, j, \ldots$ refer to the world-sheet boundary and take values $0,1, \ldots, p-1$. The coordinates of spacetime, world-sheet and world-sheet boundary are denoted by $x^{\mu}, \xi^{a}$ and $\lambda^{i}$, respectively. The corresponding metric tensors are denoted by $g_{\mu \nu}(x), \gamma_{a b}(\xi)$ and $h_{i j}(\lambda)$. The signature convention is defined by $\operatorname{diag}(-,+, \ldots,+)$, and the indices are raised by the inverse metrics $g^{\mu \nu}, \gamma^{a b}$ and $h^{i j}$.

\section{THE MULTIPOLE FORMALISM}

We begin with the covariant conservation of the fundamental matter currents - stress-energy tensor $\tau^{\mu}{ }_{\nu}$, and spin tensor $\sigma_{\mu \nu}^{\lambda}$ :

$$
\begin{gathered}
\left(D_{\nu}+\mathcal{T}^{\lambda}{ }_{\nu \lambda}\right) \tau^{\nu}{ }_{\mu}=\tau^{\nu}{ }_{\rho} \mathcal{T}^{\rho}{ }_{\mu \nu}+\frac{1}{2} \sigma^{\nu \rho \sigma} \mathcal{R}_{\rho \sigma \mu \nu} \\
\left(D_{\nu}+\mathcal{T}^{\lambda}{ }_{\nu \lambda}\right) \sigma^{\nu}{ }_{\rho \sigma}=\tau_{\rho \sigma}-\tau_{\sigma \rho} .
\end{gathered}
$$

Here, $D_{\nu}$ is the covariant derivative with the nonsymmetric connection $\Gamma^{\lambda}{ }_{\mu \nu}$, which acts on a vector $v^{\mu}$ according to the rule $D_{\nu} v^{\mu} \equiv \partial_{\nu} v^{\mu}+\Gamma^{\mu}{ }_{\lambda \nu} v^{\lambda}$. The torsion $\mathcal{T}^{\lambda}{ }_{\mu \nu}$, and curvature $\mathcal{R}^{\mu}{ }_{\nu \rho \sigma}$ are defined in the standard way:

$$
\begin{gathered}
\mathcal{T}^{\lambda}{ }_{\mu \nu} \equiv \Gamma^{\lambda}{ }_{\nu \mu}-\Gamma^{\lambda}{ }_{\mu \nu}, \\
\mathcal{R}^{\mu}{ }_{\nu \rho \sigma} \equiv \partial_{\rho} \Gamma_{\nu \sigma}^{\mu}-\partial_{\sigma} \Gamma^{\mu}{ }_{\nu \rho}+\Gamma^{\mu}{ }_{\lambda \rho} \Gamma^{\lambda}{ }_{\nu \sigma}-\Gamma^{\mu}{ }_{\lambda \sigma} \Gamma^{\lambda}{ }_{\nu \rho} .
\end{gathered}
$$

The derivative $D_{\lambda}$ is assumed to satisfy the metricity condition, $D_{\lambda} g_{\mu \nu}=0$. As a consequence, the connection $\Gamma^{\lambda}{ }_{\mu \nu}$ is split into the Levi-Civita connection $\left\{\begin{array}{c}\lambda \\ \mu \nu\end{array}\right\}$, and the contorsion $K^{\lambda}{ }_{\mu \nu}$ :

$$
\begin{gathered}
\Gamma^{\lambda}{ }_{\mu \nu}=\left\{\begin{array}{c}
\lambda \\
\mu \nu
\end{array}\right\}+K^{\lambda}{ }_{\mu \nu}, \\
K^{\lambda}{ }_{\mu \nu} \equiv-\frac{1}{2}\left(\mathcal{T}^{\lambda}{ }_{\mu \nu}-\mathcal{T}_{\nu}{ }^{\lambda}{ }_{\mu}+\mathcal{T}_{\mu \nu}{ }^{\lambda}\right) .
\end{gathered}
$$

We shall also introduce the Riemannian covariant derivative $\nabla_{\mu} \equiv D_{\mu}(\Gamma \rightarrow\{\})$, and the Riemannian curvature tensor $R^{\mu}{ }_{\nu \rho \sigma} \equiv \mathcal{R}^{\mu}{ }_{\nu \rho \sigma}(\Gamma \rightarrow\{\})$. The relation connecting the two curvature tensors reads:

$$
\mathcal{R}^{\mu}{ }_{\nu \lambda \rho}=R^{\mu}{ }_{\nu \lambda \rho}+2 \nabla_{[\lambda} K^{\mu}{ }_{\nu \rho]}+2 K^{\mu}{ }_{\sigma[\lambda} K^{\sigma}{ }_{\nu \rho]},
$$

where the indices in square brackets are antisymmetrized.

Given the system of conservation equations (1), one finds that the second one has no dynamical content. Indeed, the antisymmetric part of stress-energy tensor is completely determined by the spin tensor. One can use (1b) to eliminate $\tau^{[\mu \nu]}$ from the equation (1a), and thus obtain the conservation equation, in which only $\tau^{(\mu \nu)}$ and $\sigma^{\lambda \mu \nu}$ components appear. The resulting equation can be written in the form

$$
\begin{gathered}
\nabla_{\nu}\left(\theta^{\mu \nu}-K_{\lambda \rho}^{[\mu} \sigma^{\rho \lambda \nu]}-\frac{1}{2} K_{\lambda \rho}{ }^{[\mu} \sigma^{\nu] \rho \lambda}\right)= \\
=\frac{1}{2} \sigma_{\nu \rho \lambda} \nabla^{\mu} K^{\rho \lambda \nu},
\end{gathered}
$$

where $\theta^{\mu \nu}=\theta^{\nu \mu}$ stands for the generalized Belinfante tensor:

$$
\theta^{\mu \nu} \equiv \tau^{(\mu \nu)}-\nabla_{\rho} \sigma^{(\mu \nu) \rho}-\frac{1}{2} K_{\lambda \rho}^{(\mu} \sigma^{\nu) \rho \lambda} .
$$

The independent variables $\theta^{\mu \nu}$ and $\sigma^{\mu \nu \rho}$ are in $1-1$ correspondence with the original variables. In what follows, the conservation law in the form (2) will be the starting point for the derivation of the brane world-sheet equations.

Let us now introduce the multipole formalism, which is necessary for the derivation. It has been shown in Refs. [11, 12] that an exponentially decreasing function can be expanded into a series of $\delta$-function derivatives. For example, a tensor valued function $F^{\mu \nu}(x)$, well localized around the $(p+1)$-dimensional surface $\mathcal{M}$ in $D$ dimensional spacetime, can be decomposed in a manifestly covariant way as

$$
\begin{gathered}
F^{\mu \nu}(x)=\int_{\mathcal{M}} d^{p+1} \xi \sqrt{-\gamma}\left[M^{\mu \nu} \frac{\delta^{(D)}(x-z)}{\sqrt{-g}}-\right. \\
\left.-\nabla_{\rho}\left(M^{\mu \nu \rho} \frac{\delta^{(D)}(x-z)}{\sqrt{-g}}\right)+\cdots\right] .
\end{gathered}
$$

The surface $\mathcal{M}$ is defined by the equation $x^{\mu}=z^{\mu}(\xi)$, where $\xi^{a}$ are the surface coordinates, and the coefficients $M^{\mu \nu}(\xi), M^{\mu \nu \rho}(\xi), \ldots$ are spacetime tensors called multipole coefficients. Here, and in what follows, we shall frequently use the notion of the surface coordinate vectors

$$
u_{a}^{\mu}=\frac{\partial z^{\mu}}{\partial \xi^{a}}
$$


and the surface induced metric tensor

$$
\gamma_{a b}=g_{\mu \nu} u_{a}^{\mu} u_{b}^{\nu} .
$$

The induced metric is assumed to be nondegenerate, $\gamma \equiv \operatorname{det}\left(\gamma_{a b}\right) \neq 0$, and of Minkowski signature. The same holds for the target space metric $g_{\mu \nu}(x)$ and its determinant $g(x)$.

It has been shown in Ref. 12 that one may truncate the series in a covariant way in order to approximate the description of matter. Truncation after the leading term is called single-pole approximation, truncation after the second term is called pole-dipole approximation. The physical interpretation of these approximations is the following. In the single-pole approximation, one assumes that the brane has no thickness, which means that matter is localized on a surface. All higher approximations, including pole-dipole, allow for the non-zero thickness, and thus, for the transversal internal motion.

Apart from being covariant with respect to diffeomorphisms, the series (4) possesses two extra gauge symmetries. The first is a consequence of the fact that that there are redundant coefficients in this decomposition. Indeed, only $D-p-1$ out of $D \delta$-functions in each term of the multipole expansion (4) are effective in modeling trajectory of a brane like object in $D$-dimensional spacetime. The $p+1$ extra $\delta$-functions and the extra integrations are introduced only to covariantize the expressions. The derivatives parallel to the world-sheet are integrated out, as they should, considering the fact that matter is not localized along the brane. As a consequence, the parallel components of the higher multipole coefficients are expected to effectively disappear. It has been shown in Ref. [12] that the corresponding gauge symmetry, named extra symmetry 1 , in the pole-dipole approximation has the form

$$
\delta_{1} M^{\mu \nu}=\nabla_{a} \epsilon^{\mu \nu a}, \quad \delta_{1} M^{\mu \nu \rho}=\epsilon^{\mu \nu a} u_{a}^{\rho},
$$

where $\epsilon^{\mu \nu a}(\xi)$ are gauge parameters constrained by the boundary condition

$$
\left.n_{a} \epsilon^{\mu \nu a}\right|_{\partial \mathcal{M}}=0 .
$$

Here, $n^{a}$ is the unit boundary normal, and $\nabla_{a}$ stands for the world-sheet total covariant derivative (see the Appendix). Thus, the parallel components of $M^{\mu \nu \rho}$ transform as $\delta_{1}\left(u_{\rho}^{a} M^{\mu \nu \rho}\right)=\epsilon^{\mu \nu a}$, and can be gauged away. In fact, one can show that the parallel components of the higher multipoles are also pure gauge. In the gauge fixed multipole expansion, the only derivatives that appear are those orthogonal to the world-sheet. In the single-pole approximation, the extra symmetry 1 is trivial.

The second extra symmetry stems from the fact that the choice of the surface $x^{\mu}=z^{\mu}(\xi)$ in the expansion (4) is arbitrary. If we use another surface, let us say $x^{\mu}=z^{\prime \mu}(\xi)$, the coefficients $M^{\mu \nu}, M^{\mu \nu \rho}, \ldots$ will change to $M^{\prime \mu \nu}, M^{\prime \mu \nu \rho}, \ldots$ while leaving the function $F^{\mu \nu}(x)$ invariant. The transformation law of the $M$-coefficients, generated by the replacement $z^{\mu} \rightarrow z^{\prime \mu}$, defines the gauge symmetry that we call extra symmetry 2 .

The extra symmetry 2 is an exact symmetry of the full expansion (44), but only approximate symmetry of the truncated series. In the pole-dipole approximation, it has the form

$$
\begin{aligned}
& \delta_{2} z^{\mu}=\epsilon^{\mu} \\
& \delta_{2} M^{\mu \nu}=-M^{\mu \nu} u_{\rho}^{a} \nabla_{a} \epsilon^{\rho}-M^{\lambda \nu}\left\{\begin{array}{c}
\mu \\
\lambda \rho
\end{array}\right\} \epsilon^{\rho}-M^{\mu \lambda}\left\{\begin{array}{c}
\nu \\
\lambda \rho
\end{array}\right\} \epsilon^{\rho}, \\
& \delta_{2} M^{\mu \nu \rho}=-M^{\mu \nu} \epsilon^{\rho},
\end{aligned}
$$

provided the $M$-coefficients are subject to the hierarchy $M^{\mu \nu}=\mathcal{O}_{0}, M^{\mu \nu \rho}=\mathcal{O}_{1}$, and the free parameters $\epsilon^{\mu}(\xi)$ satisfy $\epsilon^{\mu}=\mathcal{O}_{1}$. Here, $\mathcal{O}_{n}$ stands for the order of smallness, and the condition $\epsilon^{\mu}=\mathcal{O}_{1}$ ensures that the order of truncation is not violated by the action of the symmetry transformations [12]. In the pole-dipole and higher approximations, fixing the gauge of extra symmetry 2 defines the central surface of mass. In the single-pole approximation, the extra symmetry 2 is trivial.

Now, we shall replace the general function $F^{\mu \nu}(x)$ with the stress-energy and spin tensors of the localized matter. In the pole-dipole approximation, our independent variables $\theta^{\mu \nu}$ and $\sigma^{\lambda \mu \nu}$ are written in the form

$$
\begin{gathered}
\theta^{\mu \nu}=\int d^{p+1} \xi \sqrt{-\gamma}\left[B^{\mu \nu} \frac{\delta^{(D)}(x-z)}{\sqrt{-g}}-\nabla_{\rho}\left(B^{\mu \nu \rho} \frac{\delta^{(D)}(x-z)}{\sqrt{-g}}\right)\right], \\
\sigma^{\lambda \mu \nu}=\int d^{p+1} \xi \sqrt{-\gamma} C^{\lambda \mu \nu} \frac{\delta^{(D)}(x-z)}{\sqrt{-g}}
\end{gathered}
$$

where $B^{\mu \nu}(\xi), B^{\mu \nu \rho}(\xi)$ and $C^{\lambda \mu \nu}(\xi)$ are the corresponding multipole coefficients. As we can see, the decomposition of the spin-tensor $\sigma^{\lambda \mu \nu}$ lacks the dipole term. This is because the spin is considered to be of the order of the orbital angular momentum which is already described by the dipole coefficient. Any correction to the leading term 
of (7b) would, therefore, take us beyond the pole-dipole approximation.

The symmetries of the expansion (7) are basically the same as found in the general considerations. They include spacetime and world-sheet diffeomorphisms, and two extra symmetries. The transformation properties of the multipole coefficients with respect to spacetime diffeomorphisms and world-sheet reparametrizations are determined by their index structure. Thus, $B^{\mu \nu}, B^{\mu \nu \rho}$ and $C^{\lambda \mu \nu}$ are spacetime tensors, and world-sheet scalars. As for the two extra symmetries, the transformation law of the $B$-coefficients is given by the general formulas (5) and (6), but the $C$-coefficients transform trivially:

$$
\delta_{1} C^{\lambda \mu \nu}=\delta_{2} C^{\lambda \mu \nu}=0 .
$$

This is because the expansion (7b) has a single-pole form, and we have already established that extra symmetries in the single-pole approximation are trivial. In addition, the multiple coefficients are required to obey the hierarchy $B^{\mu \nu}=\mathcal{O}_{0}, B^{\mu \nu \rho} \sim C^{\lambda \mu \nu}=\mathcal{O}_{1}$. Only then the extra symmetry 2 remains unbroken.

In what follows, we shall use the expansion (7) to solve the conservation equations (2), and define the limit of an infinitely thin brane.

\section{EQUATIONS OF MOTION}

In this section, the stress-energy and spin-tensor conservation equations (2) are analyzed in the pole-dipole approximation. The brane world-sheet equations and boundary conditions are derived in a manifestly covariant way.

\section{A. Derivation}

The brane world-sheet equations are derived in the following way. We insert (77) into (2), and solve for the unknown variables $z^{\mu}(\xi), B^{\mu \nu}(\xi), B^{\mu \nu \rho}(\xi)$ and $C^{\lambda \mu \nu}(\xi)$. The algorithm for solving this type of equation is discussed in detail in [11, 12], and here we only sketch it. The first step is to multiply the equation (2) with an arbitrary spacetime function $f_{\mu}(x)$ of compact support, and integrate over the spacetime. The resulting equation depends on the function $f_{\mu}$ and its first and second covariant derivatives, evaluated on the surface $x^{\mu}=z^{\mu}(\xi)$. Precisely, we obtain

$$
\begin{gathered}
\int d^{p+1} \xi \sqrt{-\gamma}\left[B^{\mu \nu \rho} f_{\mu ; \nu \rho}+\left(B^{\mu \nu}-D^{\mu \nu}\right) f_{\mu ; \nu}+\right. \\
\left.+\frac{1}{2} C_{\nu \rho \lambda}\left(\nabla^{\mu} K^{\rho \lambda \nu}\right) f_{\mu}\right]=0
\end{gathered}
$$

where $f_{\mu ; \nu} \equiv\left(\nabla_{\nu} f_{\mu}\right)_{x=z}, f_{\mu ; \nu \rho} \equiv\left(\nabla_{\rho} \nabla_{\nu} f_{\mu}\right)_{x=z}$, and the shorthand notation

$$
D^{\mu \nu} \equiv K_{\lambda \rho}^{[\mu} C^{\rho \lambda \nu]}+\frac{1}{2} K_{\lambda \rho}{ }^{[\mu} C^{\nu] \rho \lambda}
$$

is introduced for later convenience. Owing to the arbitrariness of the function $f_{\mu}(x)$, the terms proportional to its independent derivatives separately vanish. The independent derivatives are found by making use of the decomposition into components orthogonal and parallel to the world-sheet $x^{\mu}=z^{\mu}(\xi)$ :

$$
\begin{gathered}
f_{\mu ; \lambda}=f_{\mu \lambda}^{\perp}+u_{\lambda}^{a} \nabla_{a} f_{\mu}, \\
f_{\mu ;(\lambda \rho)}=f_{\mu \lambda \rho}^{\perp}+2 f_{\mu(\lambda a}^{\perp} u_{\rho)}^{a}+f_{\mu a b} u_{\lambda}^{a} u_{\rho}^{b}, \\
f_{\mu ;[\lambda \rho]}=\frac{1}{2} R_{\mu \lambda \rho}^{\sigma} f_{\sigma} .
\end{gathered}
$$

Here, the orthogonal and parallel components are obtained by using the projectors

$$
P_{\perp \nu}^{\mu}=\delta_{\nu}^{\mu}-u_{a}^{\mu} u_{\nu}^{a}, \quad P_{\| \nu}^{\mu}=u_{a}^{\mu} u_{\nu}^{a} .
$$

More precisely, $f_{\mu \lambda}^{\perp}=P_{\perp \lambda}^{\sigma} f_{\mu ; \sigma}, f_{\mu \lambda \rho}^{\perp}=P_{\perp \lambda}^{\sigma} P_{\perp \rho}^{\nu} f_{\mu ; \sigma \nu}$, $f_{\mu \lambda a}^{\perp}=P_{\perp \lambda}^{\sigma} u_{a}^{\nu} f_{\mu ;(\sigma \nu)}$ and $f_{\mu a b}=u_{a}^{\sigma} u_{b}^{\nu} f_{\mu ;(\sigma \nu)}$. Direct calculation yields

$$
\begin{aligned}
& f_{\mu a b}=\nabla_{(a} \nabla_{b)} f_{\mu}-\left(\nabla_{a} u_{b}^{\nu}\right) f_{\mu \nu}^{\perp} \\
& f_{\mu \rho a}^{\perp}=P_{\perp \rho}^{\nu} \nabla_{a} f_{\mu \nu}^{\perp}+\left(\nabla_{a} u_{\rho}^{b}\right) \nabla_{b} f_{\mu}+\frac{1}{2} P_{\perp \rho}{ }_{\rho}^{\lambda} u_{a}^{\nu} R_{\mu \nu \lambda}^{\sigma} f_{\sigma},
\end{aligned}
$$

which tells us that the only independent components on the surface $x^{\mu}=z^{\mu}(\xi)$ are $f_{\mu}, f_{\mu \nu}^{\perp}$ and $f_{\mu \nu \rho}^{\perp}$. We can now use (10) and (12) in the equations (9) to group the coefficients into terms proportional to the independent derivatives of $f_{\mu}$. The obtained equation has the following general structure:

$$
\begin{aligned}
& \int d^{p+1} \xi \sqrt{-\gamma}\left[X^{\mu \nu \rho} f_{\mu \nu \rho}^{\perp}+X^{\mu \nu} f_{\mu \nu}^{\perp}+X^{\mu} f_{\mu}+\right. \\
& \left.+\nabla_{a}\left(X^{\mu \nu a} f_{\mu \nu}^{\perp}+X^{\mu a b} \nabla_{b} f_{\mu}+X^{\mu a} f_{\mu}\right)\right]=0
\end{aligned}
$$

where $X$ terms are composed of various combinations of multipole coefficients $B^{\mu \nu}, B^{\mu \nu \rho}$ and $C^{\lambda \mu \nu}$, external fields $K^{\lambda}{ }_{\mu \nu}$ and $R^{\mu}{ }_{\nu \rho \sigma}$, and their derivatives. In all the expressions, the external fields are evaluated on the surface $x^{\mu}=z^{\mu}(\xi)$. Owing to the fact that $f_{\mu}, f_{\mu \nu}^{\perp}$ and $f_{\mu \nu \rho}^{\perp}$ are independent functions on the world-sheet, we deduce that the first three $X$ terms must separately vanish.

The equation $X^{\mu \nu \rho}=0$ has a simple algebraic form

$$
P_{\perp \lambda}^{(\nu} P_{\perp \rho}^{\sigma)} B^{\mu \lambda \rho}=0
$$

Its general solution is

$$
B^{\mu \nu \rho}=2 u_{a}^{(\mu} J^{\nu) \rho a}+N^{\mu \nu a} u_{a}^{\rho},
$$

where the new coefficients $J^{\mu \nu a}$ and $N^{\mu \nu a}$ are subject to the algebraic constraints

$$
J^{\mu \nu[a} u_{\nu}^{b]}=0, \quad J^{\mu \nu a}=-J^{\nu \mu a}, \quad N^{\mu \nu a}=N^{\nu \mu a} .
$$


In what follows, we shall see that the coefficients $N^{\mu \nu a}$ drop from the world-sheet equations, while $J^{\mu \nu a}$ currents couple to the background curvature.

The equations $X^{\mu \nu}=0$ and $X^{\mu}=0$ are much more complicated. The procedure goes as follows. First, we use the above decomposition of $B^{\mu \nu \rho}$ to perform a similar split of the $B^{\mu \nu}$ coefficients. As a result, new free parameters $m^{a b}(\xi)$ appear to characterize the leading term of the coefficient $B^{\mu \nu}$. Then, the equations $X^{\mu \nu}=0$ and $X^{\mu}=0$ are rewritten in terms of the undetermined parameters $m^{a b}, J^{\mu \nu a}$ and $C^{\lambda \mu \nu}$, and properly rearranged. The coefficients $N^{\mu \nu a}$ turn out to completely disappear. Skipping the details of the diagonalization procedure, which has thoroughly been demonstrated in Ref. [12], we display the resulting world-sheet equations:

$$
\begin{gathered}
P_{\perp \lambda}^{\mu} P_{\perp \rho}^{\nu}\left(\nabla_{a} J^{\lambda \rho a}+D^{\lambda \rho}\right)=0 \\
\nabla_{b}\left[m^{a b} u_{a}^{\mu}-2 u_{\lambda}^{b}\left(\nabla_{a} J^{\mu \lambda a}+D^{\mu \lambda}\right)+u_{c}^{\mu} u_{\rho}^{c} u_{\lambda}^{b}\left(\nabla_{a} J^{\rho \lambda a}+D^{\rho \lambda}\right)\right]=u_{a}^{\nu} J^{\lambda \rho a} R_{\nu \lambda \rho}^{\mu}+\frac{1}{2} C_{\nu \rho \lambda} \nabla^{\mu} K^{\rho \lambda \nu} .
\end{gathered}
$$

The world-sheet equations (15) describe the dynamics of a thick $p$-brane in $D$-dimensional spacetime with curvature and torsion. The coefficients $m^{a b}, J^{\mu \nu a}$ and $C^{\lambda \mu \nu}$ are free parameters of the theory. While $m^{a b}$ represents the effective stress-energy tensor of the brane, the $J^{\mu \nu a}$ and $C^{\lambda \mu \nu}$ currents are related to its total internal angular momentum and spin, respectively. In the particle case, our world-line equations agree with the known results in literature 6 -10].

Having solved the equations $X^{\mu \nu \rho}=X^{\mu \nu}=X^{\mu}=0$, we are left with the surface integral that vanishes itself:

$$
\int_{\partial \mathcal{M}} d^{p} \lambda \sqrt{-h} n_{a}\left(X^{\mu \nu a} f_{\mu \nu}^{\perp}+X^{\mu a b} \nabla_{b} f_{\mu}+X^{\mu a} f_{\mu}\right)=0 .
$$

The components $f_{\mu \nu}^{\perp}$ and $f_{\mu}$, when evaluated on the boundary, are mutually independent, but $\nabla_{a} f_{\mu}$ is not. This is why we decompose the $\nabla_{a}$ derivative into components orthogonal and parallel to the boundary:

$$
\nabla_{a} f_{\mu}=n_{a} \nabla_{\perp} f_{\mu}+v_{a}^{i} \nabla_{i} f_{\mu}
$$

Here, $\nabla_{\perp} \equiv n^{a} \nabla_{a}, \nabla_{i}$ is the total covariant derivative on $\partial \mathcal{M}$, and $v_{i}^{a}$ are the boundary coordinate vectors (see the Appendix for details). Now, $f_{\mu \nu}^{\perp}, \nabla_{\perp} f_{\mu}$ and $f_{\mu}$ are mutually independent, and the equation (16) yields three sets of boundary conditions:

$$
\begin{gathered}
\left.J^{\mu \nu a} n_{a} n_{\nu}\right|_{\partial \mathcal{M}}=0,\left.\quad P_{\perp \lambda}^{\mu} P_{\perp \rho}^{\nu} J^{\lambda \rho a} n_{a}\right|_{\partial \mathcal{M}}=0, \\
\left.n_{b}\left[m^{a b} u_{a}^{\mu}-2 u_{\rho}^{b}\left(\nabla_{a} J^{\mu \rho a}+D^{\mu \rho}\right)+u_{c}^{\mu} u_{\sigma}^{c} u_{\nu}^{b}\left(\nabla_{a} J^{\sigma \nu a}+D^{\sigma \nu}\right)\right]\right|_{\partial \mathcal{M}}=\left.\nabla_{i}\left(N^{i j} v_{j}^{\mu}+2 J^{\mu \nu a} n_{a} v_{\nu}^{i}\right)\right|_{\partial \mathcal{M}} .
\end{gathered}
$$

The new free parameters $N^{i j} \equiv N^{\mu \nu a} n_{a} v_{\mu}^{i} v_{\nu}^{j}$ are defined on the boundary, and appear nowhere else. This situation is familiar from the analysis of thick branes in torsionless spacetimes [12]. In fact, our world-sheet equations and boundary conditions reduce to those of Ref. [12] in the limit of vanishing torsion.

\section{B. Interpretation}

The world-sheet equations (15), and boundary conditions (18) describe the dynamics of a thick brane in
Riemann-Cartan spacetime. The free coefficients $m^{a b}$, $J^{\mu \nu a}, C^{\lambda \mu \nu}$ and $N^{i j}$ characterize the internal structure of the brane. The tensor $m^{a b}$ represents the effective $(p+1)$ dimensional stress-energy of the brane interior, while $N^{i j}$ stands for the stress-energy of the brane boundary. The coefficients $J^{\mu \nu a}$ are the world-sheet currents associated with the total internal angular momentum of the brane, and $C^{\lambda \mu \nu}$ is the spin-tensor of the constituent matter.

The dynamical equations that we have obtained differ from those of Ref. [12] by the presence of the spintorsion couplings. By inspecting their form, we realize that branes made of scalar matter can not probe space- 
time torsion. This is a generalization of the known result concerning thick particles [6 10]. We emphasize though that our predictions concerning zero-size particles disagree with the existing literature. In the next section, we shall demonstrate this in a simple example.

\section{Symmetries}

The symmetries of the world-sheet equations (15) and boundary conditions (18) are basically the same as in the case of spinless matter [12]. It is only that, in addition to $B^{\mu \nu}=\mathcal{O}_{0}, B^{\mu \nu \rho}=\mathcal{O}_{1}$, the condition $C^{\lambda \mu \nu}=\mathcal{O}_{1}$ is needed to ensure the existence of extra symmetry 2 . The transformation law of the free parameters $m^{a b}, J^{\mu \nu a}$, $C^{\lambda \mu \nu}$ and $N^{i j}$ is obtained from the known symmetry properties of the $B$ and $C$ coefficients. With respect to diffeomorphisms, the coefficients

- $m^{a b}, J^{\mu \nu a}, C^{\lambda \mu \nu}$ and $N^{i j}$ are tensors of the type defined by their index structure.

Thus, $m^{a b}$ is a world-sheet tensor, $J^{\mu \nu a}$ is a spacetime tensor and a world-sheet vector, $C^{\lambda \mu \nu}$ is a spacetime tensor, and $N^{i j}$ is a boundary tensor. The coefficients which lack certain type of indices transform as scalars under corresponding reparametrizations. For example, $J^{\mu \nu a}$ is a scalar with respect to the boundary reparametrizations, while $N^{i j}$ is a scalar with respect to spacetime and worldsheet diffeomorphisms.

The extra symmetry 1 is an algebraic symmetry, which ensures that only gauge invariant coefficients appear in properly diagonalized world-sheet equations. Using (5) and (8), we indeed find that our free parameters transform trivially:

$$
\delta_{1} m^{a b}=\delta_{1} J^{\mu \nu a}=\delta_{1} C^{\lambda \mu \nu}=\delta_{1} N^{i j}=0 .
$$

It has been shown in Ref. [12] that the peculiar $N^{i j}$ coefficients that live exclusively on the boundary are a consequence of the constraint (5b) that parameters of the extra symmetry 1 obey. If not for this, the transformation law $\delta_{1} N^{\mu \nu a}=\epsilon^{\mu \nu a}$ would imply that $N^{\mu \nu a}$ are pure gauge everywhere. Physically, the $N^{i j}$ coefficients represent a correction to the effective $p$-dimensional stressenergy tensor of the brane boundary, very much like $m^{a b}$ is $(p+1)$-dimensional effective stress-energy tensor of the brane itself. The best way to see this is to consider a brane with extra massive matter attached to its boundary. The procedure has thoroughly been demonstrated in Ref. [12], where an infinitely thin string with massive thick particles attached to its ends has been considered.

The extra symmetry 2 has been defined in Sec. III as the symmetry generated by the change of the surface $x^{\mu}=z^{\mu}(\xi)$ used in the $\delta$-function expansion. The transformation laws (6) and (8), thus obtained, can be rewritten in terms of the free coefficients $m^{a b}, J^{\mu \nu a}, C^{\lambda \mu \nu}$ and $N^{i j}$. Using the decomposition of the parameters $\epsilon^{\mu}$ into components orthogonal and parallel to the world-sheet, $\epsilon^{\mu}=\epsilon_{\perp}^{\mu}+u_{a}^{\mu} \epsilon^{a}$, we find:

$$
\begin{gathered}
\delta_{2} z^{\mu}=\epsilon_{\perp}^{\mu}+u_{a}^{\mu} \epsilon^{a}, \\
\delta_{2} m^{a b}=-\left(u_{\mu}^{c} m^{a b}+u_{\mu}^{(a} m^{b) c}\right) \nabla_{c} \epsilon_{\perp}^{\mu}-m^{b c} \nabla_{c} \epsilon^{a}-m^{a c} \nabla_{c} \epsilon^{b}+\epsilon^{c} \nabla_{c} m^{a b}, \\
\delta_{2} J^{\mu \nu a}=-m^{a b} u_{b}^{[\mu} \epsilon_{\perp}^{\nu]}, \quad \delta_{2} N^{i j}=-m^{a b} v_{a}^{i} v_{b}^{j} \epsilon^{c} n_{c}, \\
\delta_{2} C^{\lambda \mu \nu}=0 .
\end{gathered}
$$

The transformation laws (20) are used for fixing the gauge freedom of the world-sheet equations. As explained in Ref. [12], the gauge fixing of the extra symmetry 2 corresponds to the choice of the central surface of massthe surface that approximates a branelike matter distribution. In the particle case, it coincides with the usual notion of the centre of mass. It has been shown that an appropriate gauge fixing ensures that particle trajectories in flat, torsionless spacetimes coincide with straight lines. In the case of higher branes, the central surface of mass can be chosen to eliminate the boost degrees of freedom from the angular momentum charge densities $J^{\mu \nu 0}$. This is done by using the transformation law (20c). The residual extra symmetry 2 can then be used to fix the trace of the boundary stress-energy $N^{i j}$. In the string dynamics, the boundary is one-dimensional, and there is only one $N$ coefficient, which can, therefore, be completely gauged away. 


\section{Single-pole limit}

The important limit of an infinitely thin brane is obtained by discarding dipole terms in the multipole expansion (77). The resultant expression contains no $\delta$-function derivatives, and is called single-pole approximation. In our case, this is achieved by imposing the constraint $B^{\mu \nu \rho}=0$.

The consequences of the new constraint are far reaching. We can immediately write the single-pole equations by using the relation (14) that establishes 1-1 correspondence between the coefficients $B^{\mu \nu \rho}$ and the worldsheet currents $J^{\mu \nu a}$ and $N^{\mu \nu a}$. Considering the fact that $N^{i j}$ are the only surviving components of the coefficient $N^{\mu \nu a}$, the constraint $B^{\mu \nu \rho}=0$ is rewritten as

$$
J^{\mu \nu a}=0, \quad N^{i j}=0 .
$$

Substituting (21) into (15), (18), we obtain the singlepole world-sheet equations

$$
\begin{gathered}
P_{\perp \lambda}^{\mu} P_{\perp \rho}{ }_{\rho}^{\nu} D^{\lambda \rho}=0, \\
\nabla_{b}\left(m^{a b} u_{a}^{\mu}-2 u_{\lambda}^{b} D^{\mu \lambda}+u_{c}^{\mu} u_{\rho}^{c} u_{\lambda}^{b} D^{\rho \lambda}\right)=\frac{1}{2} C_{\nu \rho \lambda} \nabla^{\mu} K^{\rho \lambda \nu},
\end{gathered}
$$

and the single-pole boundary conditions

$$
\left.n_{b}\left(m^{a b} u_{a}^{\mu}-2 u_{\rho}^{b} D^{\mu \rho}+u_{c}^{\mu} u_{\sigma}^{c} u_{\nu}^{b} D^{\sigma \nu}\right)\right|_{\partial \mathcal{M}}=0 .
$$

The equations (22) and (23) describe the motion of an infinitely thin brane in Riemann-Cartan spacetime. Compared to the pole-dipole approximation, there are two striking differences. First, the coupling of the spacetime curvature to the internal angular momentum of the brane is missing. This is something one would expect to hold for spinless matter, only. Indeed, if the brane has no thickness, the transversal internal motion is not possible, and the internal orbital angular momentum vanishes [12]. The spin part of the total angular momentum, however, is expected to survive. Thus, the vanishing of the spin-curvature coupling in the single-pole limit comes as a surprise.

Another striking consequence of the single-pole limit is the algebraic nature of the precession equations (22a). This new constraint restrains the allowed forms of the spin-tensor in the presence of the background torsion. If the torsion is absent, the constraint is identically satisfied. In the next section, this unusual behaviour will be studied in the particle example.

\section{EXAMPLES}

To illustrate the relevance of the brane dynamics in Riemann-Cartan spacetimes, we shall discuss two important examples: zero-size particle and infinitely thin string. In the particle case, the novel spin-curvature coupling is shown to contradict the results of the existing literature. In the string case, the action of Refs. 15-18] is recovered, and the Kalb-Ramond antisymmetric field $\mathcal{B}^{\mu \nu}$ given a geometric interpretation.

\section{A. Particle}

The world-sheet of a particle is one-dimensional, and is called world-line. We shall parametrize it with the proper distance $\tau$, thereby fixing the reparametrization invariance:

$$
\gamma=u^{\mu} u_{\mu}=-1
$$

Here, and in what follows, the indices $a, b, \ldots$ are omitted, as they take only one value. We shall restrict to a zero-size particle in 4-dimensional spacetime. Thus, the single-pole equations (22) yield:

$$
\begin{gathered}
\nabla\left(m u^{\mu}+2 D^{\mu \nu} u_{\nu}\right)=\frac{1}{2} C_{\nu \rho \lambda} \nabla^{\mu} K^{\rho \lambda \nu}, \\
P_{\perp \lambda}^{\mu} P_{\perp \rho}^{\nu} D^{\lambda \rho}=0,
\end{gathered}
$$

where $P_{\perp}^{\mu}=\delta_{\nu}^{\mu}+u^{\mu} u_{\nu}$ is the orthogonal world-line projector. As we can see, the spin couples only to contorsion, which means that point particles follow geodesic trajectories in torsionless spacetimes. At the same time, the absence of torsion trivializes the equation (24b), and no information on the behavior of the spin-vector is available. If the background torsion is nontrivial, a geodesic deviation appears, but also a very strong constraint on the spin-vector.

Let us now apply the obtained equations to the Dirac particle. The basic property of Dirac matter is the total antisymmetry of its spin tensor $\sigma^{\lambda \mu \nu}$. As a consequence, the coefficients $C^{\lambda \mu \nu}$ are also totally antisymmetric. If we define the spin vector $s^{\mu}$ by $C^{\mu \nu \rho} \equiv e^{\mu \nu \rho \lambda} s_{\lambda}$, and the axial component of the contorsion $K^{\mu}$ as $K^{\mu} \equiv e^{\mu \nu \rho \lambda} K_{\nu \rho \lambda}$, where $e^{\mu \nu \rho \sigma}$ is the covariant totally antisymmetric LeviCivita tensor, the equations (24) become

$$
\begin{gathered}
\nabla\left(m u^{\mu}+K^{[\mu} s^{\nu]} u_{\nu}\right)+\frac{1}{2} s^{\nu} \nabla^{\mu} K_{\nu}=0, \\
K_{\perp}^{[\mu} s_{\perp}^{\nu]}=0 .
\end{gathered}
$$

The equation (25b) implies that the orthogonal component of $s^{\mu}$ always orients itself along the background direction $K_{\perp}^{\mu}$. This unusual behavior suggests the possibility that the spin itself might vanish in the zero-size limit. Moreover, the same suggestion comes from the disappearance of the total angular momentum $J^{\mu \nu}$ in the singlepole approximation. Indeed, the zero-size limit naturally rules out the orbital part of the angular momentum. If 
$J^{\mu \nu}$ is seen as the sum of orbital and spin parts, its disappearance inevitably implies the disappearance of the spin itself.

To justify this scenario, the authors of Refs. [26, 27] have analyzed the wave packet solutions of the flat space Dirac equation. The idea was to check if the wave packets could be viewed as zero-size objects. For that purpose, the wave packet size $\ell$, and its wavelength $\lambda$ are considered in the limit $\ell \rightarrow 0, \lambda / \ell \rightarrow 0$. It has been discovered that the wave packet spin and orbital angular momentum disappear simultaneously in this limit. Thus, the spin vector $s^{\mu}$ vanishes in the single-pole approximation, and the particle trajectory becomes a geodesic line even in the presence of torsion.

The single-pole results obtained in this section disagree with the results found in literature [7-9]. In these early approaches, the antisymmetric part of the stress-energy tensor $\tau^{[\mu \nu]}$ has been treated as an independent variable, in spite of the restriction (1b). This imposed unnecessary constraints on $\sigma^{\lambda \mu \nu}$. In particular, the spin of the Dirac particle was ruled out. In our approach, the only independent variables are the spin-tensor $\sigma^{\lambda \mu \nu}$ and the symmetric Belinfante tensor $\theta^{\mu \nu}$. As a result, our single-pole limit is less restrictive, and allows an equal treatment of all massive elementary fields.

\section{B. String}

The string trajectory is a two-dimensional world-sheet with one-dimensional boundary. As in the particle case, the boundary line will be parametrized with the proper distance $\tau$, and the indices $i, j, \ldots$, which take only one value, will be omitted. Thus, the boundary metric $h$, and the tangent vector $v^{a}$ satisfy

$$
h=v^{a} v_{a}=-1 .
$$

The only peculiarity of the string dynamics, as compared to higher branes, is the possibility to gauge away the $N^{i j}$ coefficients. Indeed, there is only one such component in the string case, and one free parameter in the transformation law (20c). After fixing the gauge $N=0$, the $\epsilon^{a}$ part of the extra symmetry 2 reduces to reparametrizations.

In what follows, we shall consider infinitely thin strings, and thus, make use of the single-pole equations (22) and (23). In this approximation, the extra symmetries are trivial, and the coefficients $J^{\mu \nu a}$ and $N$ are zero. The remaining coefficients $m^{a b}$ and $C^{\rho \mu \nu}$ carry the information on the type of matter the string is made of.

Our idea is to try and find the string type whose classical dynamics coincides with that of Refs. 15 -18]. There, the string is coupled to the Kalb-Ramond antisymmetric field $\mathcal{B}_{\mu \nu}$, commonly interpreted as the torsion potential, $K_{\mu \nu \rho} \propto \nabla_{\mu} \mathcal{B}_{\nu \rho}+\nabla_{\nu} \mathcal{B}_{\rho \mu}+\nabla_{\rho} \mathcal{B}_{\mu \nu}$ [19 25]. If we accept this picture, however, we find that no choice of the coefficients $m^{a b}$ and $C^{\rho \mu \nu}$ leads to the satisfactory solution. Indeed, the spin-torsion couplings in the world-sheet equations (22) contain torsion derivatives, which do not exist in the string dynamics of Refs. 1518]. The l.h.s. contains only derivatives parallel to the world-sheet, which can not compensate for the orthogonal derivatives on the r.h.s. The only way to get rid of these is to have $C_{\rho \mu \nu} \propto u_{\rho}^{a} u_{\mu}^{b} u_{\nu}^{c}$, but the assumed total antisymmetry of $K^{\mu \nu \rho}$ rules out this choice. Thus, whatever choice of $C^{\rho \mu \nu}$ is made, the resulting wordsheet equations will contain undesirable couplings to torsion derivatives. We are led to the conclusion that the usual interpretation of $\mathcal{B}^{\mu \nu}$ as the torsion potential is not supported by the classical string dynamics in RiemannCartan spacetimes.

In what follows, we shall continue searching for the string type that realizes the correct $\mathcal{B}^{\mu \nu}$ coupling, whatever geometric interpretation of $\mathcal{B}^{\mu \nu}$ field may be. Skipping the details of our pursuit, we shall describe the scheme that we have found to work.

First, we restrict our attention to spacetimes characterized by the contorsion of the form

$$
K^{\mu \nu \rho}=K^{\mu \nu} K^{\rho},
$$

where $K^{\mu \nu} \equiv-K^{\nu \mu}$ is an arbitrary antisymmetric tensor, and $K^{\rho}$ is an arbitrary vector field. It is obvious that this decomposition is not unique. Indeed, the transformation $K^{\mu \nu} \rightarrow \alpha K^{\mu \nu}, K^{\rho} \rightarrow \alpha^{-1} K^{\rho}$ leaves the contorsion $K^{\mu \nu \rho}$ invariant. Using this freedom, we shall fix the norm of the vector field $K^{\rho}$ to be

$$
K^{\rho} K_{\rho}=\kappa
$$

where $\kappa=1,-1$ or 0 , depending on whether $K^{\rho}$ is spacelike, timelike or lightlike vector. From now on, we shall assume that $\kappa$ takes only one value in the whole spacetime.

Our second assumption concerns the spin tensor coefficients $C^{\rho \mu \nu}$. We specify their form by the relation

$$
C^{\rho \mu \nu}=s K^{\rho} u^{\mu \nu},
$$

where $u^{\mu \nu} \equiv e^{a b} u_{a}^{\mu} u_{b}^{\nu}$, and $s$ is a constant that measures the spin magnitude.

With these assumptions, the world-sheet equations (22), and boundary conditions (23) are rewritten in terms of the free coefficients $m^{a b}$, and the external fields $K^{\mu \nu}$. First, we calculate the $D^{\mu \nu}$ tensor, and find that it reduces to

$$
D^{\mu \nu}=s \kappa e^{a b} u_{a}^{[\mu} K^{\nu]},
$$

where $K^{\mu a} \equiv K^{\mu \nu} u_{\nu}^{a}$. The precession equations (22a) are then automatically satisfied, and we are left with the world-sheet equations (22b), and boundary conditions (23). Now, we calculate the right-hand side of (22b), and find

$$
C_{\nu \rho \lambda} \nabla^{\mu} K^{\rho \lambda \nu}=s \kappa\left(F^{\mu \rho \lambda} u_{\rho \lambda}-2 e_{a b} \nabla^{a} K^{b \mu}\right),
$$

with

$$
F^{\mu \nu \rho} \equiv \nabla^{\mu} K^{\nu \rho}+\nabla^{\nu} K^{\rho \mu}+\nabla^{\rho} K^{\mu \nu} .
$$


With the help of these expressions, the world-sheet equations are rewritten as

$$
\nabla_{b}\left(\bar{m}^{a b} u_{a}^{\mu}\right)=\frac{s \kappa}{2} F^{\mu \rho \lambda} u_{\rho \lambda},
$$

where the new parameters $\bar{m}^{a b}$ are related to $m^{a b}$ as follows:

$$
\bar{m}^{a b} \equiv m^{a b}-\frac{s \kappa}{2} \gamma^{a b} K^{\mu \nu} u_{\mu \nu} .
$$

Using the total antisymmetry of the $F^{\mu \rho \lambda}$ coefficients in (28), the new coefficients $\bar{m}^{a b}$ are shown to be covariantly conserved,

$$
\nabla_{b} \bar{m}^{a b}=0 .
$$

This means that Nambu-Goto matter is allowed as the constituent matter of our string. Indeed, by demanding $\bar{m}^{a b}=T \gamma^{a b}$, where $T$ is a constant commonly interpreted as the string tension, the condition (29) is automatically satisfied. At the same time, we obtain the world-sheet equations in their final form

$$
\nabla_{a} u^{a \mu}=\frac{s \kappa}{2 T} F^{\mu \rho \lambda} u_{\rho \lambda} .
$$

Following the same procedure, the boundary conditions are rewritten as

$$
\left.n^{a}\left(u_{a}^{\mu}+\frac{s \kappa}{T} K^{\mu b} e_{a b}\right)\right|_{\partial \mathcal{M}}=0 .
$$

The world-sheet equations (30), and boundary conditions (31) are exactly the same as obtained by varying the string action of Refs. [15 18. This action describes a string interacting with an additional external antisymmetric field $\mathcal{B}_{\mu \nu}(x)$, and has the form

$$
S=T \int d^{2} \xi \sqrt{-\gamma}\left[g_{\mu \nu}(x) u_{a}^{\mu} u_{b}^{\nu} \gamma^{a b}+\mathcal{B}_{\mu \nu}(x) u_{a}^{\mu} u_{b}^{\nu} e^{a b}\right],
$$

where the world-sheet metric $\gamma_{a b}$ is considered as an independent variable. One can verify that it is indeed minimized by our equations (30) and (31), provided the identification

$$
\mathcal{B}^{\mu \nu} \equiv \frac{s \kappa}{T} K^{\mu \nu}
$$

is made. Thus, the Kalb-Ramond antisymmetric field $\mathcal{B}^{\mu \nu}$ is recognized as a part of the spacetime torsion.

Note that the geometric interpretation found in literature is not the same. There, the external field $\mathcal{B}^{\mu \nu}$ has commonly been treated as a torsion potential, rather than the torsion itself. The authors of Refs. 19 23] studied the influence of the string background fields on the string dynamics, and succeeded in rewriting the worldsheet equations in geometric terms. In this setting, the field strength of the $\mathcal{B}^{\mu \nu}$ field turned out to define the torsion part of the modified geometry. We must emphasize, however, that this new geometry is characterized by the presence two connections, and does not belong to the class of Riemann-Cartan geometries considered in this paper.

In Ref. 24], a similar line of reasoning has been applied to the string low-energy effective action. This action governs the dynamics of the string background fieldsthe spacetime metric $g_{\mu \nu}$, Kalb-Ramond field $\mathcal{B}_{\mu \nu}$ and the dilaton $\Phi$. It was shown that the field strength of the Kalb-Ramond field could be absorbed in the antisymmetric part of a new connection. In this approach, however, the derived torsion is free, in the sense that no string-torsion coupling is specified. Thus, this approach is complementary to our treatment of strings in fixed backgrounds, with no background dynamics specified.

Finally, let us mention that the same discussion applies to the pure geometric considerations of Ref. [25].

In summary, there are different ways of relating the $\mathcal{B}_{\mu \nu}$ field to torsion, and our result follows from the particular approach of treating probe strings in RiemannCartan backgrounds.

\section{CONCLUDING REMARKS}

In this paper, we have analyzed classical dynamics of brane like objects in backgrounds of nontrivial geometry. In particular, our target space is characterized by both, curvature and torsion. The type of matter fields the brane is made of has not been specified. We have only assumed that matter fields are sharply localized around a brane.

The method we have used is a generalization of the Mathisson-Papapetrou method for pointlike matter [1, 2]. It has already been used in Refs. [11, 12] for the study of strings and higher branes in Riemannian spacetimes. In this work, we have extended the analysis to RiemannCartan spacetimes.

Our exposition is summarized as follows. In section $\amalg$, we have defined the conservation law of the stress-energy and spin tensors, and eliminated the antisymmetric part of stress-energy by noticing that it is not an independent variable. Thereby, the conservation equations are rewritten in terms of the spin tensor $\sigma^{\lambda \mu \nu}$ and the symmetric Belinfante tensor $\theta^{\mu \nu}$. A brief recapitulation of the covariant multipole formalism, and its symmetry properties has been given by invoking the results of Ref. [12]. Then, the pole-dipole approximation has been defined for the independent variables, only.

In section III, the brane world-sheet equations and boundary conditions have been obtained in a manifestly covariant form. In the particle case, the pole-dipole result has been shown to agree with the results of the existing literature. The single-pole limits, however, turned out to differ. This is a consequence of the fact that earlier approaches incorrectly treated the antisymmetric part of the stress-energy $\tau^{[\mu \nu]}$ as an independent variable.

In Section IV] we have analyzed the 0-brane and 1brane examples. In the particle case, the world-line equations have been compared to the known pole-dipole equa- 
tions [8, [9]. They are found to coincide in the pole-dipole approximation, but have different single-pole limits. In the string case, the world-sheet equations are analyzed in the limit of zero thickness. By an appropriate choice of the spin-tensor and the background torsion, we have recovered the string action of Refs. [15 18]. In particular, the Kalb-Ramond antisymmetric tensor field $\mathcal{B}^{\mu \nu}$ has been recognized as the torsion itself, rather than its potential. The apparent contradiction with the existing literature on the subject is illusive. There are different ways of relating the $\mathcal{B}_{\mu \nu}$ field to torsion, and they are often complementary to each other. While we treat strings coupled to fixed Riemann-Cartan backgrounds, some authors consider dynamical backgrounds with no string couplings 24, 25], or employ non Riemann-Cartan geometries [19 23].

\section{Acknowledgments}

This work is partly supported by the Serbian Ministry of Science and Technological Development, under contract No. 141036. One of the authors (Marko Vojinović) acknowledges hospitality at the Institute for $\mathrm{Nu}$ clear Research and Nuclear Energy in Sofia (Bulgaria) during his visit as an early stage researcher, supported by the FP6 Marie Curie Research Training Network "ForcesUniverse" MRTN-CT-2004-005104.

\section{Appendix: Differential geometry of surfaces}

In this work, we deal with the geometry of surfaces embedded in Riemann-Cartan spacetime. Let us summarize the basic notions and relations used throughout the paper.

We shall consider a $D$-dimensional Riemann-Cartan spacetime parametrized by the coordinates $x^{\mu}$. Its metric tensor is denoted by $g_{\mu \nu}(x)$, and is assumed to have Minkowski signature. Given the metric, one defines the Levi-Civita connection

$$
\left\{\begin{array}{c}
\mu \\
\rho \sigma
\end{array}\right\} \equiv \frac{1}{2} g^{\mu \lambda}\left(\partial_{\rho} g_{\lambda \sigma}+\partial_{\sigma} g_{\lambda \rho}-\partial_{\lambda} g_{\rho \sigma}\right),
$$

and the Riemannian covariant derivative $\nabla_{\lambda}$ :

$$
\nabla_{\lambda} V^{\mu} \equiv \partial_{\lambda} V^{\mu}+\left\{\begin{array}{c}
\mu \\
\rho \lambda
\end{array}\right\} V^{\rho}
$$

We now introduce a $(p+1)$-dimensional surface $\mathcal{M}$ parametrized by the coordinates $\xi^{a}$. If the surface equation is $x^{\mu}=z^{\mu}(\xi)$, one can define the coordinate vectors

$$
u_{a}^{\mu} \equiv \frac{\partial z^{\mu}}{\partial \xi^{a}},
$$

and the induced metric tensor

$$
\gamma_{a b} \equiv g_{\mu \nu}(z) u_{a}^{\mu} u_{b}^{\nu} .
$$

The surface $\mathcal{M}$ is assumed to be everywhere regular, and the coordinates $x^{\mu}$ and $\xi^{a}$ well defined. If it is future directed, the induced metric has Minkowski signature. We can now define the total Riemannian covariant derivative $\nabla_{a}$, that acts on both types of indices:

$$
\nabla_{a} V^{\mu b}=\partial_{a} V^{\mu b}+\left\{\begin{array}{c}
\mu \\
\lambda \rho
\end{array}\right\} u_{a}^{\rho} V^{\lambda b}+\left\{\begin{array}{c}
b \\
c a
\end{array}\right\} V^{\mu c} .
$$

Here, $\left\{\begin{array}{c}a \\ b c\end{array}\right\}$ is the Levi-Civita connection on the surface, so that the metricity conditions $\nabla_{a} g_{\mu \nu}=\nabla_{a} \gamma_{b c}=0$ are identically satisfied.

A spacetime vector $V^{\mu}$ can uniquely be split into vectors orthogonal and tangential to the surface by using the projectors

$$
P_{\| \nu}^{\mu} \equiv u_{a}^{\mu} u_{\nu}^{a}, \quad P_{\perp \nu}^{\mu} \equiv \delta_{\nu}^{\mu}-u_{a}^{\mu} u_{\nu}^{a} .
$$

Thus, $V^{\mu}=V_{\perp}^{\mu}+V_{\|}^{\mu}$, where $V_{\perp}^{\mu} \equiv P_{\perp}^{\mu} V^{\nu}$, and $V_{\|}^{\mu} \equiv$ $P_{\| \nu}^{\mu} V^{\nu}$.

The surface $\mathcal{M}$ may have a boundary $\partial \mathcal{M}$, and we denote its coordinates by $\lambda^{i}$. The boundary is assumed to satisfy the analogous geometric requirements as the surface itself. Given the boundary $\xi^{a}=\zeta^{a}(\lambda)$, one introduces its coordinate vectors

$$
v_{i}^{a} \equiv \frac{\partial \zeta^{a}}{\partial \lambda^{i}},
$$

and the induced metric

$$
h_{i j}=\gamma_{a b}(\zeta) v_{i}^{a} v_{j}^{b} .
$$

The boundary connection is defined to be the Levi-Civita connection $\left\{\begin{array}{c}i \\ j k\end{array}\right\}$, so that the total covariant derivative $\nabla_{i}$, which acts as

$\nabla_{i} V^{\mu b j}=\partial_{i} V^{\mu b j}+\left\{\begin{array}{c}\mu \\ \lambda \rho\end{array}\right\} v_{i}^{\rho} V^{\lambda b j}+\left\{\begin{array}{c}b \\ c a\end{array}\right\} v_{i}^{a} V^{\mu c j}+\left\{\begin{array}{c}j \\ k i\end{array}\right\} V^{\mu b k}$,

satisfies the metricity conditions $\nabla_{i} g_{\mu \nu}=\nabla_{i} \gamma_{a b}=$ $\nabla_{i} h_{j k}=0$. Here, $v_{i}^{\mu} \equiv u_{a}^{\mu} v_{i}^{a}$ are the spacetime components of the boundary coordinate vectors. The boundary projectors are defined as $p_{\|}^{\mu} \equiv v_{i}^{\mu} v_{\nu}^{i}$ and $p_{\perp}^{\mu} \equiv \delta_{\nu}^{\mu}-v_{i}^{\mu} v_{\nu}^{i}$.

Throughout the paper, the covariant form of the Stokes theorem is used:

$$
\int_{\mathcal{M}} d^{p+1} \xi \sqrt{-\gamma} \nabla_{a} V^{a}=\int_{\partial \mathcal{M}} d^{p} \lambda \sqrt{-h} n_{a} V^{a} .
$$

Here, $n_{a}$ is the normal to the boundary. It is defined as

$$
n_{a}=\frac{1}{p !} e_{a b_{1} \ldots b_{p}} e^{i_{1} \ldots i_{p}} v_{i_{1}}^{b_{1}} \ldots v_{i_{p}}^{b_{p}}
$$

where $e_{a b_{1} \ldots b_{p}}$ and $e^{i_{1} \ldots i_{p}}$ are totally antisymmetric world tensors on the surface and the boundary, respectively. They are defined using the Levi-Civita symbols $\varepsilon_{a b_{1} \ldots b_{p}}$ and $\varepsilon^{i_{1} \ldots i_{p}}$, and corresponding metric determinants:

$$
e_{a b_{1} \ldots b_{p}}(\xi) \equiv \sqrt{-\gamma} \varepsilon_{a b_{1} \ldots b_{p}}, \quad e^{i_{1} \ldots i_{p}}(\lambda) \equiv \frac{1}{\sqrt{-h}} \varepsilon^{i_{1} \ldots i_{p}}
$$

The normal $n_{a}$ is always spacelike, and satisfies the following identities:

$$
n_{a} n^{a}=1, \quad n_{a} v_{i}^{a}=0, \quad P_{\perp \nu}^{\mu}=p_{\perp \nu}^{\mu}-n^{\mu} n_{\nu},
$$

where $n^{\mu} \equiv u_{a}^{\mu} n^{a}$. 
[1] M. Mathisson, Acta. Phys. Pol. 6, 163 (1937).

[2] A. Papapetrou, Proc. R. Soc. A 209, 248 (1951).

[3] W. Tulczyjew, Acta. Phys. Pol. 18, 393 (1959).

[4] A. H. Taub, J. Math. Phys. 5, 112 (1964).

[5] G. Dixon, Nuovo Cimento 38, 1616 (1965); 34, 317 (1964); Proc. R. Soc. London 314, 499 (1970); 319, 509 (1970); Gen. Relativ. Gravit. 4, 199 (1973).

[6] A. Trautman, Bull. Acad. Pol. Sci. Ser. Sci. Math. Astron. Phys. 20, 895 (1972).

[7] F. W. Hehl, Phys. Lett. A 36, 225 (1971).

[8] P. B. Yasskin and W. R. Stoeger, Phys. Rev. D 21, 2081 (1980).

[9] K. Nomura, T. Shirafuji, and K. Hayashi, Prog. Theor. Phys. 86, 1239 (1991).

[10] K. Nomura, T. Shirafuji, and K. Hayashi, Prog. Theor. Phys. 87, 1275 (1992).

[11] M. Vasilić and M. Vojinović, Phys. Rev. D 73, 124013 (2006).

[12] M. Vasilić and M. Vojinović, J. High Energy Phys. 07, 028 (2007).

[13] Y. Nambu, Phys. Rev. D 10, 4262 (1974).

[14] T. Goto, Prog. Theor. Phys. 46, 1560 (1971).

[15] M. B. Green, J. H. Schwarz, and E. Witten, Superstring
Theory (Cambridge University Press, 1987).

[16] E. S. Fradkin and A. A. Tseytlin, Phys. Lett. B 158, 316 (1985); Nucl. Phys. B261, 1 (1985).

[17] C. G. Callan, D. Friedan, E. J. Martinec, and M. J. Perry, Nucl. Phys. B262, 593 (1985).

[18] T. Banks, D. Nemeschansky, and A. Sen, Nucl. Phys. B277, 67 (1986).

[19] J. Scherk and J. H. Schwarz, Phys. Lett. B 52, 347 (1974); Nucl. Phys. B81, 118 (1974).

[20] T. Dereli and R. W. Tucker, Class. Quant. Grav. 12, L31 (1995).

[21] T. L. Curtright and C. K. Zachos, Phys. Rev. Lett. 53, 1799 (1984).

[22] S. Mukhi, Phys. Lett. B 162, 345 (1985).

[23] B. Sazdovic, Mod. Phys. Lett. A 20, 897 (2005); Int. J. Mod. Phys. A 20, 5501 (2005).

[24] R. I. Nepomechie, Phys. Rev. D 32, 3201 (1985).

[25] P. G. O. Freund and R. I. Nepomechie, Nucl. Phys. B 199, 482 (1982).

[26] M. Vasilić and M. Vojinović, arXiv:0806.3228.

[27] M. Vasilić and M. Vojinović, J. High Energy Phys. 08, 104 (2008). 